\title{
Predictors of Bone Mineral Density Improvement after Parathyroidectomy for Secondary Hyperparathyroidism: A Retrospective Single-Center Analysis
}

\author{
Manabu Okada ${ }^{1}$ (1) $\cdot$ Yoshihiro Tominaga $^{1} \cdot$ Toshihide Tomosugi $^{1} \cdot$ Takahisa Hiramitsu $^{1} \cdot$ \\ Toshihiro Ichimori ${ }^{1} \cdot$ Tetsuhiko Sato $^{2}$
}

\begin{abstract}
Background Parathyroidectomy (PTx) reportedly increases bone mineral density (BMD) in patients with severe secondary hyperparathyroidism (SHPT). To date, however, there has not been sufficient evidence on predictors of BMD improvement post-PTx for SHPT, an issue the present retrospective cohort study aimed to address.

Methods A total of 173 SHPT patients who underwent total PTx with forearm autograft between 2009 and 2017 were included in the present study. Demographic information, perioperative laboratory data and pre- and post-PTx BMD values (measured by dual-energy X-ray absorptiometry) were collected from their medical records. The change in BMD post-PTx in the lumbar spine was evaluated as the primary outcome. Then, a multivariate logistic regression analysis was performed for $\mathrm{a} \geq 10 \%$ increase in BMD post-PTx.

Results Overall, the median BMD in the lumbar spine was increased by $8.7 \%$ post-PTx. The multivariate logistic regression analysis revealed that age $\geq 70$ years $(P=0.005$; odds ratio [OR], 0.138 ; 95\% confidence interval [CI]: 0.034-0.555), serum Ca level ( $P=0.017$; OR, 0.598 ; 95\% CI: 0.392-0.911) and pre-PTx BMD in the lumbar spine $(P=0.003$; OR, 0.013 ; 95\% CI: 0.001-0.229) were negatively associated with a $\geq 10 \%$ increase in BMD post-PTx. Conclusion Our study demonstrated that presurgical age, serum Ca levels and BMD values could better predict an improvement in BMD post-PTx in SHPT patients.
\end{abstract}

\section{Introduction}

The risk of fracture in patients with chronic kidney disease (CKD) is significantly higher than that in the general population $[1,2]$. Some of the known risk factors for

Supplementary Information The online version contains supplementary material available at https://doi.org/10.1007/s00268021-06186-1.

Manabu Okada

ubanam@nagoya2.jrc.or.jp

1 Department of Transplantation and Endocrine Surgery, Nagoya Daini Red Cross Hospital, 2-9 Myoken-cho, Showaku, Nagoya 4668650, Japan

2 Department of Endocrinology, Nagoya Daini Red Cross Hospital, Nagoya, Japan fracture in CKD patients include older age, diabetes, female sex and secondary hyperparathyroidism (SHPT) which is commonly found in CKD patients as a cause of renal osteodystrophy [3, 4]. The mechanisms whereby SHPT increases the risk of fracture mainly involve osteitis fibrosa and osteoporosis following persistently elevated parathyroid hormone (PTH) levels [5-8].

Parathyroidectomy (PTx) has been reported to reduce the risk of fracture and increase bone mineral density (BMD) in patients with severe SHPT and remains the most effective treatment option for advanced SHPT [9, 10]. However, although some SHPT patients experience a significant increase in BMD post-PTx, others do not achieve marked improvement in their BMD.

A few studies have reported some predictors of postPTx BMD changes [11-13]. However, the validity of the 
multivariate analyses conducted in these studies is hampered by the relatively small sample sizes. To help identify potential predictors of PTx-induced improvement in BMD, we conducted the present retrospective study with a much larger population of SHPT patients who underwent total PTx with forearm autograft.

\section{Material and methods}

\section{Study design and subjects}

All patients who underwent PTx for SHPT at Nagoya Daini Red Cross Hospital (Nagoya, Japan) between January 2009 and December 2017 were retrospectively analyzed. Patients who had their initial PTx at other institutions, missing BMD data, persistent SHPT post-PTx (intact PTH levels $\geq 60 \mathrm{pg} / \mathrm{mL}$ on the first day post-PTx) and renal transplantation within 1-year post-PTx were excluded from the present study. For the patients who met the inclusion criteria, data on demographic and clinical characteristics, such as age, sex, dialysis vintage, renal diagnosis, perioperative laboratory findings, pre- and post-PTx BMD and pre- and post-PTx T-scores were collected from their electronic medical records. Preoperative blood sampling tests were performed at the first visit of the patients to the Department of Endocrine Surgery at Nagoya Daini Red Cross Hospital within 3 months before PTx. In the present study, the percent change in BMD of the lumbar spine was evaluated as the primary outcome. The improvement in BMD was defined as a postoperative BMD increase of $\geq 10 \%$. To estimate predictive factors for BMD improvement induced by PTx, patients were divided into two groups according to post-PTx BMD change in the lumbar spine as follows: I-BMD group, comprising of patients with $a \geq 10 \%$ increase in BMD and NI-BMD group, comprising of patients with $a<10 \%$ increase in BMD. The abovementioned patient characteristics and laboratory data were compared between the two groups, followed by multivariate analyses. The present study was approved by the Institutional Review Board of Nagoya Daini Red Cross Hospital and was conducted according to the Declaration of Helsinki. Consent for participation could not be obtained from each patient, given the retrospective nature of the present study. Instead, the study protocol and supporting contents are available on the webpage of Nagoya Daini Red Cross Hospital. The opportunity and rights of the patients to drop out of the study were guaranteed.

\section{Surgical procedure and postoperative management}

The routine operative technique was total PTx with forearm autograft, which involved resection of the thymic tongue followed by insertion of approximately $90 \mathrm{mg}$ of the parathyroid tissue into the forearm muscles. Surgical indications for SHPT were established according to the clinical practice guidelines issued by the Japanese Society for Dialysis Therapy as follows [14]: high PTH levels (intact $\mathrm{PTH}, \geq 500 \mathrm{pg} / \mathrm{mL}$ ), hyperphosphatemia (serum phosphorus $[\mathrm{sP}], \geq 6.0 \mathrm{mg} / \mathrm{dL}$ ), or hypercalcemia (serum calcium $[\mathrm{sCa}], \geq 10.0 \mathrm{mg} / \mathrm{dL}$ ) when refractory to medical treatment and severe osteopenia, pathological fractures, renal stones, parathyroid gland diameter of $>10 \mathrm{~mm}$ as measured on imaging and severe SHPT-related symptoms. After PTx, calcium (Ca) and vitamin D supplementation with oral $\mathrm{Ca}$ carbonate $1.5-12 \mathrm{~g} / \mathrm{day}$ and alfacalcidol 1-3 $\mu \mathrm{g} /$ day was initiated when the sCa level decreased to $<9 \mathrm{mg} / \mathrm{dL}$. In cases with severe hypocalcemia or tetany, intravenous $\mathrm{Ca}$ was also administered. The doses of the $\mathrm{Ca}$ and vitamin $\mathrm{D}$ supplementation were adjusted to maintain the sCa levels within a range of $8.0-9.0 \mathrm{mg} / \mathrm{dL}$. After discharge, $\mathrm{Ca}$ and vitamin $\mathrm{D}$ supplementation was continued at optimal doses at dialysis clinics. Evaluation using dual-energy X-ray absorptiometry (DEXA) was scheduled 1-year post-PTx, and low BMD (T-score, $<-2.5$ ) was considered diagnostic for osteoporosis.

\section{Measurements}

BMD values were measured by DEXA (QDR DiscoveryA; Hologic Inc., Tokyo, Japan). In the measurement of lumbar spine, the L2-L4 vertebrae were laterally scanned, with the mean of these three measurements determined as the lumbar spine BMD. Anteroposterior DEXA of the lumbar spine was not included in the assessment to avoid the influence of calcification of the descending aorta. Preand post-PTx BMD values were calculated within 6 months before PTx and 1-year post-PTx, respectively. $\mathrm{T}$-scores represented the numbers of standard deviations from the mean young healthy adult's BMD. Intact PTH levels were measured by an enzyme immunoassay (TOSOH Company, Tokyo, Japan). The sCa and sP levels were measured using the standard methods. When the serum albumin levels were $<4.0 \mathrm{mg} / \mathrm{dL}$, the sCa levels were corrected as follows [15]:

Corrected $\mathrm{Ca}(\mathrm{mg} / \mathrm{dL})=$ measured $\mathrm{sCa}(\mathrm{mg} / \mathrm{dL})-$ albumin $(\mathrm{g} / \mathrm{dL})+4.0$

The percent change in BMD post-PTx was calculated as follows:

Percent change of BMD $(\%)=$ (post-PTx BMD $[\mathrm{g} /$ $\left.\mathrm{cm}^{2}\right]-$ pre-PTx BMD $\left.\left[\mathrm{g} / \mathrm{cm}^{2}\right]\right) \times 100 /$ pre-PTx BMD $(\mathrm{g} /$ $\mathrm{cm}^{2}$ ). 
Fig. 1 Study selection

flowchart. $B M D$, bone mineral density; I-BMD, bone mineral density increased by $10 \%$ or $N I-B M D$, bone mineral density increased by less than $10 \%$ after parathyroidectomy; $P T x$, parathyroidectomy more after parathyroidectomy;

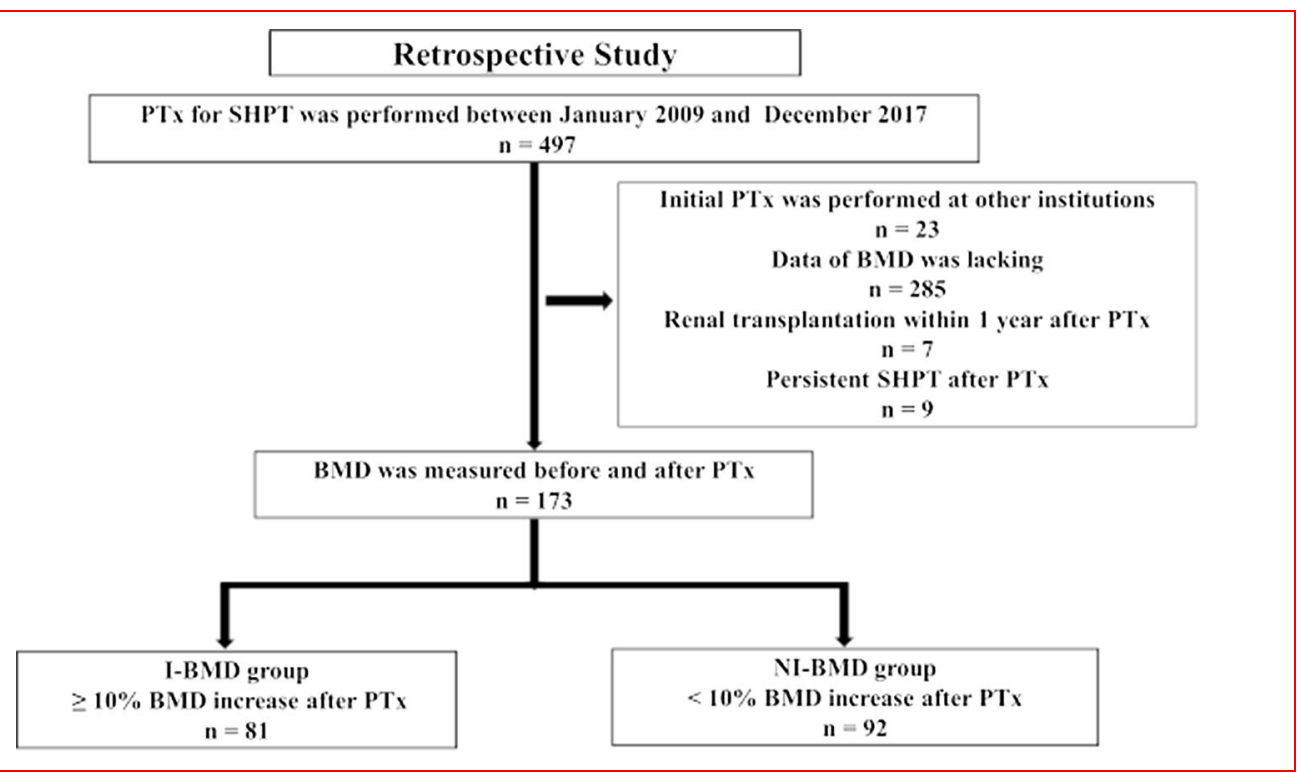

\section{Statistical analysis}

Nominal variables were analyzed using Pearson's chisquared test and the Mann-Whitney $U$ test was applied to compare continuous and ordinal variables. All results were presented as median and interquartile range. The Spearman rank correlation coefficient was used to evaluate the correlation among the variables (Electronic Supplementary Material [ESM] 1). We conducted a multivariate stepwise logistic regression analysis for the $\geq 10 \%$ increase in BMD in the lumbar spine post-PTx. In addition, the crude and multivariable-adjusted odds ratios (ORs) for the $\geq 10 \%$ increase in BMD in the lumbar spine for the categories of pre-PTx sCa levels and BMD values were also examined using logistic regression analysis. Furthermore, a multivariate linear regression analysis of the ratio of postPTx BMD to pre-PTx BMD (post-PTx BMD-to-pre-PTx BMD ratio) in the lumbar spine, including other explanatory variables, was also performed to assess the robustness of the results of the logistic regression analysis (ESM 2). Logarithmic conversion was applied to the values of PTH, bone-specific alkaline phosphatase (BAP) and post-PTx BMD-to-pre-PTx BMD ratio for normal distribution in the linear regression. The percent change in BMD was not adopted as the objective variable because the percent change can take a negative value, which cannot be converted to logarithms ( $\mathrm{Log})$. Age was treated as a categorical variable in 10-year increments. SPSS version 23.0 for Windows (IBM Japan Ltd., Tokyo, Japan) and EZR version 1.40 for Windows [16] were used for statistical analyses. Two-tailed $P$ values $<0.05$ were considered statistically significant.

\section{Theory and calculation}

In the present study, the BMD change in the lumbar spine rather than the radius was chosen as the primary outcome because the radius is a cortical bone-rich tissue that is likely to have less BMD improvement after PTx than the lumbar spine [11-13].

The rationale for defining a $10 \%$ or greater increase in $\mathrm{BMD}$ in the lumbar spine as a significant improvement in bone mass is based on the following evidence: Lu et al. reported a $12.2 \%$ increase in $\mathrm{BMD}$ of the lumbar spine 1 year after PTx [12]. Sharma also pointed out that the BMD change in the lumbar spine was $12.3 \%$ approximately 2 years after PTx [17]. By contrast, a 6\%-9\% increase in lumbar BMD induced by bisphosphonate has been reported to reduce the vertebral fracture risk by over $40 \%$ in a few years $[18,19]$. Taken together, we ended up with the notion that a $10 \%$ or greater BMD increase is meaningful because DEXA values are known to fluctuate by about several percent [20].

\section{Results and discussion}

\section{Preoperative characteristics}

Figure 1 illustrates that 173 patients met the inclusion criteria and were included in the study. Of the 173 patients, 81 were assigned to the I-BMD group (comprising patients with $\mathrm{a} \geq 10 \%$ increase in BMD) and 92 were assigned to the NI-BMD group (comprising patients with $a<10 \%$ increase in BMD). All the patients, except three were undergoing dialysis. Two patients (with forearm and jaw fractures, respectively) in the I-BMD group and three 
Table 1 Patient characteristics before PTx

\begin{tabular}{|c|c|c|c|c|}
\hline & $\begin{array}{l}\text { All patients } \\
(\mathrm{n}=173)\end{array}$ & $\begin{array}{l}\text { NI-BMD } \\
(\mathrm{n}=92)\end{array}$ & $\begin{array}{l}\text { I-BMD } \\
(\mathrm{n}=81)\end{array}$ & $P$ value \\
\hline Age (years) & $58(47-63)$ & $58(47-64)$ & $57(47-63)$ & .534 \\
\hline Sex (male/female) & $81 / 92$ & $42 / 50$ & $39 / 42$ & .861 \\
\hline $\mathrm{HD} / \mathrm{PD}$ & $161 / 9$ & $87 / 4$ & $74 / 5$ & .827 \\
\hline Dialysis vintage (months) & $117(76-170)$ & $116(88-172)$ & $120(66-161)$ & .430 \\
\hline $\mathrm{BMI}\left(\mathrm{kg} / \mathrm{m}^{2}\right)$ & $21.5(19.4-24.4)$ & $21.8(20.0-24.6)$ & $20.9(18.9-23.8)$ & .164 \\
\hline History of fracture (\%) & $5(2.9)$ & $3(3.3)$ & $2(2.5)$ & 1.000 \\
\hline Renal diagnosis $(\%)$ & & & & .526 \\
\hline Glomerulonephritis & $101(58.4)$ & $53(57.6)$ & $48(59.3)$ & \\
\hline Diabetic nephropathy & $26(15.0)$ & $13(14.1)$ & $13(16.0)$ & \\
\hline Congenital/hereditary disease & $11(6.4)$ & $7(7.6)$ & $4(4.9)$ & \\
\hline Hypertension & $17(9.8)$ & $9(9.8)$ & 8 ( 9.9) & \\
\hline Others & $5(2.9)$ & $1(1.1)$ & $4(4.9)$ & \\
\hline Unknown & $13(7.5)$ & $9(9.8)$ & $4(4.9)$ & \\
\hline \multicolumn{5}{|l|}{ Laboratory data } \\
\hline $\mathrm{Alb}(\mathrm{g} / \mathrm{dL})$ & $4.3(4.0-4.5)$ & $4.3(4.0-4.5)$ & $4.2(4.0-4.5)$ & .733 \\
\hline Corrected Ca (mg/dL) & $10.2(9.5-10.7)$ & $10.4(9.8-11.0)$ & $9.9(9.2-10.5)$ & .002 \\
\hline $\mathrm{P}(\mathrm{mg} / \mathrm{dL})$ & $5.0(4.0-6.1)$ & $4.7(3.8-6.1)$ & $5.1(4.2-6.1)$ & .145 \\
\hline i-PTH (pg/mL) & $476.5(260.0-846.8)$ & $456.0(259.0-808.0)$ & $547.0(269.5-930.0)$ & .262 \\
\hline $\mathrm{BAP}(\mu \mathrm{g} / \mathrm{L})$ & $28.6(18.8-43.6)$ & $23.1(17.2-36.5)$ & $32.3(23.7-54.9)$ & .002 \\
\hline $1,25-\mathrm{OHD}(\mathrm{pg} / \mathrm{mL})$ & $23.7(11.4-38.7)$ & $21.3(13.0-39.3)$ & $23.8(11.4-38.4)$ & .693 \\
\hline BMD in the lumbar spine $\left(\mathrm{g} / \mathrm{cm}^{2}\right)$ & $0.63(0.56-0.74)$ & $0.65(0.57-0.75)$ & $0.61(0.52-0.72)$ & .077 \\
\hline T-score at the lumbar spine & $-2.7(-3.8$ to -1.2$)$ & $-2.5(-3.3$ to -1.0$)$ & $-3.0(-3.9$ to -1.6$)$ & .047 \\
\hline BMD in the radius $\left(\mathrm{g} / \mathrm{cm}^{2}\right)$ & $0.44(0.38-0.52)$ & $0.47(0.37-0.52)$ & $0.42(0.38-0.49)$ & .101 \\
\hline $\mathrm{T}$-score at the radius & $-3.5(-4.6$ to -2.5$)$ & $-3.2(-4.3$ to -2.0$)$ & $-3.7(-4.8$ to -2.9$)$ & .019 \\
\hline \multicolumn{5}{|l|}{ Medical therapy } \\
\hline Cinacalcet HCL (mg/day) & & & & .243 \\
\hline 0 & $73(42.2)$ & $44(47.8)$ & $29(35.8)$ & \\
\hline$<50$ & $40(23.1)$ & $18(19.6)$ & $22(27.2)$ & \\
\hline$\geq 50$ & $60(34.7)$ & $30(32.6)$ & $30(37.0)$ & \\
\hline VDRA $(\%)$ & $133(76.9)$ & $71(77.2)$ & $62(76.5)$ & .922 \\
\hline Bisphosphonate (\%) & $3(1.7)$ & $1(1.1)$ & $2(2.5)$ & .911 \\
\hline Corticosteroid (\%) & $24(13.9)$ & $18(19.6)$ & $6(7.4)$ & .037 \\
\hline
\end{tabular}

$P$ compares the NI-BMD and I-BMD groups

Data for continuous variables are presented as median (interquartile range)

1,25-OHD, 1,25-dihydroxy vitamin D; Alb, albumin; $B A P$, bone-specific alkaline phosphatase; $B M D$, bone mineral density; $B M I$, body mass index; $\mathrm{Ca}$, calcium; Cinacalcet $\mathrm{HCl}$, cinacalcet hydrochloride; $H D$, hemodialysis; $I-B M D$, bone mineral density increased by $\geq 10 \%$ after parathyroidectomy; $i-P T H$, intact parathyroid hormone; NI-BMD, bone mineral density increased by $<10 \%$ after parathyroidectomy; $P$, phosphorus; $P D$, peritoneal dialysis; $P T x$, parathyroidectomy; VDRA, vitamin D receptor activator

patients (with lumbar spine, hip and forearm fractures, respectively) in the NI-BMD group had a history of fracture prior to PTx. Significant differences in sCa level (9.9 vs. $10.4 \mathrm{mg} / \mathrm{dL}, P=0.002)$, BAP (32.3 vs. $23.3 \mu \mathrm{g} / \mathrm{L}$, $P=0.002), \mathrm{T}$-score at the lumbar spine and radius $(-3.0$ vs. $-2.5, P=0.047$ and -3.7 vs. $-3.2, \quad P=0.019$, respectively) and the proportion of the patients treated with corticosteroid (7.4 vs. $19.6 \%, P=0.037)$ were found between the I-BMD and NI-BMD groups (Table 1). The other baseline characteristics did not differ between the two groups. None of the variables had an absolute correlation coefficient greater than 0.7 (ESM1). 
Table 2 Postoperative outcomes

\begin{tabular}{|c|c|c|c|c|}
\hline & $\begin{array}{l}\text { All patients } \\
(\mathrm{n}=173)\end{array}$ & $\begin{array}{l}\text { NI-BMD } \\
(n=92)\end{array}$ & $\begin{array}{l}\text { I-BMD } \\
(\mathrm{n}=81)\end{array}$ & $P$-value \\
\hline Number of parathyroid glands resected at PTx & $4(4-4)$ & $4(4-4)$ & $4(4-4)$ & .430 \\
\hline Total weight of the parathyroid glands (mg) & $1396(900-2079)$ & $1444(887-1828)$ & $1321(933-2196)$ & .781 \\
\hline Min-PTH (pg/mL) & $5.0(3.0-10.0)$ & $5.0(3.0-8.0)$ & $5.0(4.0-11.0)$ & .538 \\
\hline $\mathrm{Ca}$ on the first day post-PTx $(\mathrm{mg} / \mathrm{dL})$ & $8.8(8.2-9.2)$ & $8.9(8.5-9.3)$ & $8.4(9.0-8.9)$ & .001 \\
\hline Ca supplementation at discharge (mg/day) & $1200(600-2400)$ & $1200(600-1800)$ & $1200(600-2400)$ & .065 \\
\hline Alfacalcidol at discharge $(\mu \mathrm{g} /$ day $)$ & $1.00(1.00-1.75)$ & $1.00(1.00-1.00)$ & $1.00(1.00-2.00)$ & .096 \\
\hline i-PTH at 1 -year post-PTx $(\mathrm{pg} / \mathrm{mL})$ & $29.5(15.8-63.3)$ & $48.0(21.8-71.3)$ & $21.0(12.0-46.3)$ & $<.001$ \\
\hline Post-PTx BMD in the lumbar spine $\left(\mathrm{g} / \mathrm{cm}^{2}\right)$ & $0.68(0.61-0.81)$ & $0.64(0.58-0.73)$ & $0.73(0.66-0.90)$ & $<.001$ \\
\hline Post-PTx T-score at the lumbar spine & $\begin{array}{l}-2.1(-3.0 \\
\text { to }-0.4)\end{array}$ & $\begin{array}{l}-2.4(-3.5 \\
\text { to }-1.5)\end{array}$ & $-1.4(-2.5-1.0)$ & $<.001$ \\
\hline Percent change in BMD in the lumbar spine (\%) & $8.7(-0.2-18.0)$ & $0.7(-5.5-5.9)$ & $19.0(14.4-26.8)$ & $<.001$ \\
\hline Post-PTx BMD in the radius $\left(\mathrm{g} / \mathrm{cm}^{2}\right)$ & $0.45(0.39-0.52)$ & $0.47(0.9-0.52)$ & $0.43(0.38-0.50)$ & .249 \\
\hline Post-PTx T-score at the radius & $\begin{array}{l}-3.3(-4.3 \\
\text { to }-2.3)\end{array}$ & $\begin{array}{l}-3.1(-4.1 \\
\text { to }-2.0)\end{array}$ & $\begin{array}{l}-3.6(-4.6 \\
\text { to }-2.6)\end{array}$ & .080 \\
\hline Percent change in BMD in the radius $(\%)$ & $1.3(-0.7-3.7)$ & $1.0(-1.5-3.0)$ & $2.2(0.5-4.4)$ & .034 \\
\hline $\begin{array}{l}\text { Time from PTx to post-PTx BMD examination } \\
\text { (months) }\end{array}$ & $12(11-13)$ & $12(11-13)$ & $12(11-13)$ & .586 \\
\hline Any fracture after PTx $(\%)$ & $4(2.3)$ & $2(2.2)$ & $2(2.5)$ & 1.000 \\
\hline Follow-up after PTx (months) & $40(25-73)$ & $38(24-78)$ & $46(25-64)$ & .815 \\
\hline
\end{tabular}

$P$ compares the NI-BMD and I-BMD groups

Data for the continuous variables are presented as median (interquartile range)

$B M D$, bone mineral density; $I-B M D$, bone mineral density increased by $10 \%$ or more after parathyroidectomy; $i-P T H$, intact parathyroid hormone; Min-PTH, parathyroid hormone on the first day after parathyroidectomy; NI-BMD, bone mineral density increased by less than $10 \%$ after parathyroidectomy; $P T x$, parathyroidectomy

\section{Postoperative outcomes}

Overall, at 1-year post-PTx, the median BMD in the lumbar spine was increased by $8.7 \%$. The I-BMD group achieved a significant improvement in lumbar T-score post-PTx ( -3.0 before PTx to -1.4 in 1-year post-PTx). None of the patients received any additional treatment for osteoporosis within 1-year post-PTx but received vitamin $\mathrm{D}$ and $\mathrm{Ca}$ supplementation. The $\mathrm{sCa}$ levels on the first day post-PTx $(8.4$ vs. $8.9 \mathrm{mg} / \mathrm{dL}, P=0.001)$ and the intact PTH 1-year post-PTx $(21.0$ vs. $48.0 \mathrm{pg} / \mathrm{mL}, P<0.001)$ were significantly lower in the I-BMD group than in the NI-BMD group (Table 2). In the lumbar spine, post-PTx BMD $\left(0.73\right.$ vs. $\left.0.64 \mathrm{~g} / \mathrm{cm}^{2}, P<0.001\right)$, T-score $(-1.4$ vs. $-2.4, P<0.001)$ and percent change of BMD (19.0 vs $0.7 \%, P<0.001)$ were significantly higher in the I-BMD group than in the NI-BMD group (Table 2).

\section{Logistic regression analyses}

The univariate logistic regression analysis demonstrated that pre-PTx sCa $(P=0.003$; OR, $0.572 ; 95 \%$ confidence interval [CI]: 0.397-0.824), $\log$ pre-PTx BAP $(P=0.013$; OR, 1.695; 95\% CI: 1.117-2.572) and treatment with corticosteroid $\quad(P=0.026 ; \quad$ OR, $0.329 ; \quad 95 \% \quad \mathrm{CI}$ : $0.124-0.875)$ had significant associations with $\geq 10 \%$ increase in BMD post-PTx. The multivariate logistic regression analysis revealed that age $\geq 70$ years ( $P=0.005$; OR, 0.138; 95\% CI: 0.034-0.555), pre-PTx sCa $(P=0.017$; OR, 0.598; 95\% CI: 0.392-0.911), prePTx BMD in the lumbar spine $(P=0.003$; OR, $0.013 ; 95 \%$ CI: $0.001-0.229)$ and corticosteroid use $(P=0.002$; OR, 0.153 ; 95\% CI: $0.045-0.514)$ were negatively associated with $\geq 10 \%$ increase in BMD post-PTx (Table 3 ). Figure 2 shows the unadjusted and multivariable-adjusted ORs for $\geq 10 \%$ increase in BMD post-PTx associated with the categories of pre-PTx sCa and BMD values. The multivariate adjusted OR of $\geq 10 \%$ increase in BMD significantly decreased when the $\mathrm{sCa}$ levels were within the range of $10.0-10.4 \mathrm{mg} / \mathrm{dL}$ (OR, 0.319; 95\% CI: 0.112-0.912) and $\geq 10.5 \mathrm{mg} / \mathrm{dL} \quad(\mathrm{OR}, 0.343 ; 95 \%$ CI: $0.127-0.927$; Fig. 2). Similarly, a downhill relationship was observed with a significant decrease in the multivariate adjusted OR of $\geq 10 \%$ increase in BMD, with pre-PTx BMD values of 
Table 3 Logistic regression for $\geq 10 \%$ increases in BMD in the lumbar spine post-PTx

\begin{tabular}{|c|c|c|c|c|c|c|}
\hline \multirow[t]{2}{*}{ Factors } & \multicolumn{3}{|c|}{ Univariate } & \multicolumn{3}{|c|}{ Multivariate } \\
\hline & OR & $95 \% \mathrm{CI}$ & $P$ value & OR & $95 \% \mathrm{CI}$ & $P$ value \\
\hline \multicolumn{7}{|l|}{ Age (years, reference to $<50$ years) } \\
\hline $50-59$ & 1.364 & $0.629-2.955$ & .432 & 0.812 & $0.328-2.011$ & .652 \\
\hline $60-69$ & 1.013 & $0.461-2.226$ & .974 & 0.457 & $0.172-1.215$ & .117 \\
\hline$\geq 70$ & 0.492 & $0.150-1.615$ & .242 & 0.138 & $0.034-0.555$ & .005 \\
\hline Male sex & 1.105 & $0.608-2.011$ & .743 & & & \\
\hline Peritoneal dialysis & 1.470 & $0.381-5.674$ & .576 & & & \\
\hline Dialysis vintage (months) & 0.999 & $0.995-1.003$ & .485 & & & \\
\hline BMI $\left(\mathrm{kg} / \mathrm{m}^{2}\right)$ & 0.951 & $0.871-1.037$ & .255 & 0.907 & $0.814-1.011$ & .077 \\
\hline Diabetes & 1.162 & $0.504-2.676$ & .725 & & & \\
\hline History of fracture & 0.751 & $0.122-4.610$ & .757 & & & \\
\hline Pre-PTx Alb (g/dL) & 0.910 & $0.405-2.047$ & .820 & & & \\
\hline Pre-PTx corrected Ca (mg/dL) & 0.572 & $0.397-0.824$ & .003 & 0.598 & $0.392-0.911$ & .017 \\
\hline Pre-PTx P (mg/dL) & 1.161 & $0.967-1.394$ & .110 & & & \\
\hline Log pre-PTx i-PTH (pg/mL) & 1.266 & $0.814-1.970$ & .295 & & & \\
\hline Log pre-PTx BAP $(\mu \mathrm{g} / \mathrm{L})$ & 1.695 & $1.117-2.572$ & .013 & 1.570 & $0.967-2.549$ & .068 \\
\hline Pre-PTx BMD in the lumbar spine $\left(\mathrm{g} / \mathrm{cm}^{2}\right)$ & 0.162 & $0.021-1.257$ & .082 & 0.013 & $0.001-0.229$ & .003 \\
\hline \multicolumn{7}{|l|}{ Cinacalcet HCL (mg/day, reference to $0 \mathrm{mg}$ /day) } \\
\hline$<50$ & 1.854 & $0.850-4.044$ & .121 & & & \\
\hline$\geq 50$ & 1.517 & $0.761-3.024$ & .236 & & & \\
\hline Vitamin D receptor activator & 0.965 & $0.475-1.959$ & .922 & & & \\
\hline Bisphosphonate & 2.304 & $0.205-25.890$ & .499 & & & \\
\hline Corticosteroid & 0.329 & $0.124-0.875$ & .026 & 0.153 & $0.045-0.514$ & .002 \\
\hline
\end{tabular}

$A l b$, albumin; $B A P$, bone-specific alkaline phosphatase; $B M D$, bone mineral density; $B M I$, body mass index; $C a$, Calcium; $95 \% C I, 95 \%$ confidence interval; Cinacalcet $\mathrm{HCl}$, cinacalcet hydrochloride; $i$ - $P T H$, intact parathyroid hormone; Log, logarithm; $O R$, odds ratio; $P$, phosphorus; PTx, parathyroidectomy

$0.55-0.64 \mathrm{~g} / \mathrm{cm}^{2} \quad$ (OR, 0.349; 95\% CI: $\left.0.124-0.979\right)$, $0.65-0.74 \mathrm{~g} / \mathrm{cm}^{2}$ (OR, 0.302; 95\% CI: 0.094-0.970) and $\geq 0.75 \mathrm{~g} / \mathrm{cm}^{2}$ (OR, 0.203; 95\% CI: 0.061-0.670; Fig. 2).

\section{Discussion}

Increase in BMD post-PTx probably contributes to the reduction in fracture risk among patients with advanced SHPT [9, 10, 21, 22]. We retrospectively examined 173 SHPT patients to clarify the predictive factors for post-PTx BMD improvement. In the present study, a multivariate logistic regression analysis revealed that age, sCa level and pre-PTx BMD are possible predictors of BMD improvement post-PTx in SHPT patients. The multivariate analysis also revealed that corticosteroid treatment was negatively associated with post-PTx BMD changes. The results obtained from the logistic regression analyses were also supported by those of the multivariate linear regression analysis of post-PTx BMD-to-pre-PTx BMD ratio in the lumbar spine (ESM 2). Some previous studies also reported BMD changes after PTx for SHPT. In a study with 15
SHPT patients, Yano et al. reported PTH and ALP levels as predictors of post-PTx BMD changes [11]. Another study with 26 SHPT patients by Lu et al. showed that BMD changes correlated negatively with BAP and correlated positively with tartrate-resistant acid phosphatase type $5 \mathrm{~b}$ [12]. Moreover, Li et al. conducted a study in 34 SHPT patients, which demonstrated that parathyroid weight and pre-PTx BMD could be used to predict BMD changes [13]. However, the sample sizes in these studies were too small for a multivariate analysis. Conversely, several studies with relatively large sample sizes have focused on post-PTx BMD changes in patients with primary hyperparathyroidism (PHPT) [17, 23]. Sharma et al. demonstrated that age, sex and pre-PTx BMD had a significant association with BMD changes post-PTx in 123 PHPT patients [17]. Furthermore, a correlation between pre-PTx sCa and postPTx BMD changes was reported by Lee et al. in a study with 92 PHPT patients [23].

In the present study, elderly patients exhibited a poor improvement in post-PTx BMD, which is consistent with the report by Sharma et al. [17]. Since physical activity has 

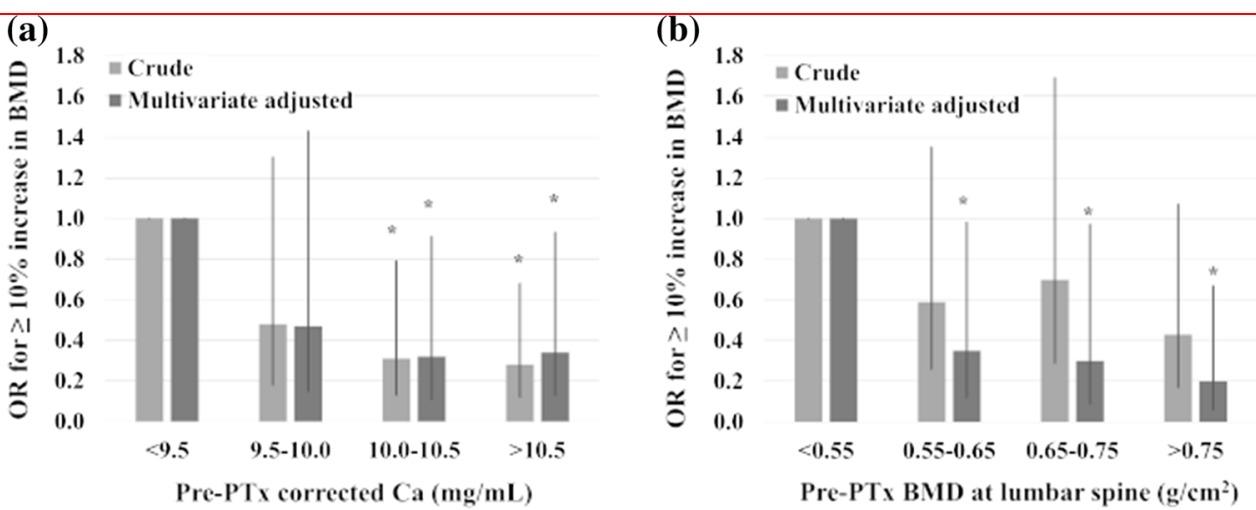

Fig. 2 ORs and 95\% CIs for $\geq 10 \%$ increase in BMD in the lumbar spine according to the categories of pre-PTx sCa levels and pre-PTx BMD in the lumbar spine using crude and multivariable-adjusted logistic models. a ORs according to the categories of pre-PTx sCa levels. The multivariable-adjusted analysis included age, BMI, Log BAP, pre-PTx BMD in the lumbar spine and treatment with corticosteroid. b ORs according to categories of pre-PTx BMD in the lumbar spine. The multivariable-adjusted analysis included age, BMI, Log BAP, pre-PTx sCa and treatment with corticosteroid. $* P<0.05$. $B A P$, bone-specific alkaline phosphatase; $B M D$, bone mineral density; $B M I$, body mass index; $95 \% C I$, 95\% confidence interval; $\log$, logarithm; $O R$, odds ratio; $P T x$, parathyroidectomy; $s C a$, serum calcium

been linked to increased bone mass in osteoporotic patients [24], it seems likely to hypothesize that the decline in physical activity with age might have hindered BMD improvement in our elderly subjects. In addition, aging itself reportedly decreased bone turnover [25].

To the best of our knowledge, the significant association observed herein between lower pre-PTx sCa levels and post-PTx BMD improvement in SHPT patients is a novel finding. In the present study, the lower sCa levels in the I-BMD group may reflect the extent of $\mathrm{Ca}$ adsorption onto bones [26]. In addition, lower pre-PTx sCa level probably increases the frequency of post-PTx hypocalcemia and doses of $\mathrm{Ca}$ and vitamin $\mathrm{D}$ supplementation, which may in turn contribute to enhanced bone mass. Furthermore, supplementation with a higher dose of vitamin D post-PTx might contribute to not only the extent in BMD change but also the difference in post-PTx PTH levels between the I-BMD and NI-BMD groups because of the known suppressive effect of vitamin D on PTH [27].

The association we established between lower pre-PTx BMD values and greater post-PTx BMD increase rates concurs with some previous reports $[7,14,28]$. The results of these studies serve as strong evidence supporting PTx in SHPT patients with bone loss. Although calcimimetics are effective in improving BMD, a past randomized study has shown PTx to be superior to calcimimetics on this front [29]. Therefore, PTx could be considered for SHPT patients with osteoporosis, even those with well-controlled biochemical parameters.

The major limitations of the present study were its single-center retrospective nature and potential selection bias arising from the study design. Furthermore, data on bone metabolism markers other than BAP were not obtained. In addition, there was a lack in long-term data on $\mathrm{Ca}$ and vitamin D supplementation and patients' daily activities after surgery. Further studies with larger sample sizes and longer observation periods are expected to validate the findings of our study.

In conclusion, age ( $\geq 70$ years) could negatively impact post-PTx bone density improvements whereas presurgical lower $\mathrm{Ca}$ levels and bone mineral density could predict enhancements in bone mass a year after PTx. Earlier surgical intervention should be considered for patients with advanced hyperparathyroidism and marked osteopenia and is expected to achieve positive outcomes in terms of bone mass in patients without hypercalcemia.

Acknowledgements The authors thank Ms. Momo Kamiya at the Division of Medical Statistics at Nagoya Daini Red Cross Hospital for valuable data collection and Enago (www.enago.jp) for the English language review.

\section{Declarations}

Conflicts of interest The authors declare that they have no conflicts of interest.

Human rights The study protocol was approved by the Institutional Review Board of Nagoya Daini Red Cross Hospital, and the study was conducted in accordance with the Declaration of Helsinki.

Informed consent Informed consent for participation could not be obtained from all individual participants, owing to the retrospective nature of the present study. Instead, the study protocol and supporting contents are published on the webpage of Nagoya Daini Red Cross Hospital. The opportunity and rights of the patients to opt out from the study were guaranteed.

Open Access This article is licensed under a Creative Commons Attribution 4.0 International License, which permits use, sharing, adaptation, distribution and reproduction in any medium or format, as 
long as you give appropriate credit to the original author(s) and the source, provide a link to the Creative Commons licence, and indicate if changes were made. The images or other third party material in this article are included in the article's Creative Commons licence, unless indicated otherwise in a credit line to the material. If material is not included in the article's Creative Commons licence and your intended use is not permitted by statutory regulation or exceeds the permitted use, you will need to obtain permission directly from the copyright holder. To view a copy of this licence, visit http://creativecommons. org/licenses/by/4.0/.

\section{References}

1. Alem AM, Sherrard DJ, Gillen DL et al (2000) Increased risk of hip fracture among patients with end-stage renal disease. Kidney Int 58:396-399

2. Wakasugi M, Kazama JJ, Taniguchi M et al (2013) Increased risk of hip fracture among Japanese hemodialysis patients. J Bone Miner Metab 31:315-321

3. Jadoul M, Albert JM, Akiba T et al (2006) Incidence and risk factors for hip or other bone fractures among hemodialysis patients in the Dialysis Outcomes and Practice Patterns Study. Kidney Int 70:1358-1366

4. Danese MD, Kim J, Doan QV et al (2006) PTH and the Risks for Hip, Vertebral and Pelvic Fractures Among Patients on Dialysis. Am J Kidney Dis 47:149-156

5. Drüeke TB, Massy ZA (2016) Changing bone patterns with progression of chronic kidney disease. Kidney Int 89:289-302

6. Fukagawa M, Kazama JJ, Kurokawa K (2002) Renal osteodystrophy and secondary hyperparathyroidism. Nephrol Dial Transplant 17(suppl 10):2-5

7. Neves KR, Graciolli FG, dos Reis LM et al (2007) Vascular calcification: Contribution of parathyroid hormone in renal failure. Kidney Int 71:1262-1270

8. Uzawa T, Hori M, Ejiri S et al (1995) Comparison of the effects of intermittent and continuous administration of human parathyroid hormone (1-34) on rat bone. Bone 16:477-484

9. Rudser KD, de Boer IH, Dooley A et al (2007) Fracture Risk after Parathyroidectomy among Chronic Hemodialysis Patients. J Am Soc Nephrol 18:2401-2407

10. Isaksson E, Ivarsson K, Akaberi S et al (2017) The effect of parathyroidectomy on risk of hip fracture in secondary hyperparathyroidism. World J Surg 41:2304-2311

11. Yano S, Sugimoto $T$, Tsukamoto $T$ et al (2003) Effect of parathyroidectomy on bone mineral density in hemodialysis patients with secondary hyperparathyroidism: possible usefulness of preoperative determination of parathyroid hormone level for prediction of bone regain. Horm Metab Res 35:259-264

12. Lu KC, Ma WY, Yu JC et al (2012) Bone turnover markers predict changes in bone mineral density after parathyroidectomy in patients with renal hyperparathyroidism. Clin Endocrinol 76:634-642

13. Fang Li WuJ, Luo J et al (2018) Changes in bone mineral density after total parathyroidectomy without autotransplantation in the end-stage renal disease patients with secondary hyperparathyroidism. BMC Nephrol 19(1):142
14. Guideline Working Group, Japanese Society for Dialysis therapy (2008) Clinical practice guideline for the management of secondary hyperparathyroidism. Ther Apher Dial 6:514-525

15. Payne RB, Little AJ, Williams RB et al (1973) Interpretation of serum calcium in patients with abnormal serum proteins. Br Med J 15:643-646

16. Kanda Y (2013) Investigation of the freely available easy-to-use software "EZR" for medical statistics. Bone Marrow Transplant 48:452-458

17. Sharma J, Itum DS, Moss L et al (2014) Predictors of Bone Mineral Density Improvement in Patients Undergoing Parathyroidectomy for Primary Hyperparathyroidism. World J Surg 38:1268-1273

18. Black DM, Cummings SR, Karpf DB et al (1996) Randomised trial of effect of alendronate on risk of fracture in women with existing vertebral fractures. Fracture Intervention Trial Research Group Lancet 348:1535-1541

19. Liberman UA, Weiss SR, Bröll J et al (1995) Effect of oral alendronate on bone mineral density and the incidence of fractures in postmenopausal osteoporosis. The Alendronate Phase III Osteoporosis Treatment Study Group. N Engl J Med 333:1437-1443

20. Larnach TA, Boyd SJ, Smart RC et al (1992) Reproducibility of lateral spine scans using dual energy X-ray absorptiometry. Calcif Tissue Int 51:255-258

21. Abdelhadi M, Nordenström J (1998) Bone Mineral Recovery after Parathyroidectomy in Patients with Primary and Renal Hyperparathyroidism. J Clin Endocrinol Metab 83:3845-3851

22. Chou FF, Lee CH, Lee CT (1999) Muscle Force and Bone Mineral Density after Parathyroidectomy and Subcutaneous Autotransplantation for Secondary Hyperparathyroidism. World J Surg 23:452-457

23. Lee D, Walker MD, Chen HY et al (2019) Bone mineral density changes after parathyroidectomy are dependent on biochemical profile. Surgery 165:107-113

24. Benedetti MG, Furlini G, Zati A et al (2018) The Effectiveness of Physical Exercise on Bone Density in Osteoporotic Patients. Biomed Res Int 2018:4840531

25. Kyvernitakis I, Saeger U, Ziller V et al (2013) The Effect of Age, Sex Hormones and Bone Turnover Markers on Calcaneal Quantitative Ultrasonometry in Healthy German Men. J Clin Densitom 16:320-328

26. Jain N, Reilly RF (2017) Hungry bone syndrome: Curr Opin Nephrol Hypertens 26:250-255

27. Hamdy NA, Kanis JA, Beneton MN et al (1995) Effect of alfacalcidol on natural course of renal bone disease in mild to moderate renal failure. BMJ 310:358-363

28. Chou FF, Chen JB, Lee $\mathrm{CH}$ et al (2001) Parathyroidectomy can improve bone mineral density in patients with symptomatic secondary hyperparathyroidism. Arch Surg 136:1064

29. Cruzado JM, Moreno P, Torregrosa JV et al (2016) A randomized study comparing parathyroidectomy with cinacalcet for treating hypercalcemia in kidney allograft recipients with hyperparathyroidism. J Am Soc Nephrol 27:2487-2494

Publisher's Note Springer Nature remains neutral with regard to jurisdictional claims in published maps and institutional affiliations. 